EPJ Web of Conferences 113,04005 (2016)

DOI: $10.1051 /$ epjconf/201611304005

(C) Owned by the authors, published by EDP Sciences, 2016

\title{
Recent Deuteron Compton Scattering Results and Extracted Neutron Polarizabilities
}

\author{
L.S. Myers ${ }^{1,2, a}$ \\ ${ }^{1}$ Thomas Jefferson National Accelerator Facility, Newport News, VA 23606, USA \\ ${ }^{2}$ Presented on behalf of the COMPTON@MAX-lab collaboration
}

\begin{abstract}
The COMPTON@MAX-lab collaboration has recently published a new measurement of elastic photon scattering from deuterium using tagged photons at the MAX IV Laboratory [1]. The experiment utilized the Tagged Photon Facility at MAX IV and three of the largest $\mathrm{NaI}(\mathrm{Tl})$ detectors in the world. Correction terms to the cross section were determined via Monte Carlo simulations [2,3] and were confirmed by comparisons to the well-known ${ }^{12} \mathrm{C}(\gamma, \gamma){ }^{12} \mathrm{C}$ reaction [4]. These results represent the most extensive data on deuteron Compton scattering ever measured and effectively double the world data set. In addition, the energy range overlaps previous experiments and extends nearly $20 \mathrm{MeV}$ higher where the sensitivity to the polarizabilities is enhanced. As a result, we have obtained the neutron polarizabilities as $\alpha_{n}=[11.55 \pm 1.25$ (stat) \pm 0.2 (BSR) \pm $0.8($ th $)] \times 10^{-4} \mathrm{fm}^{3}$ and $\beta_{n}=[3.65 \mp 1.25$ (stat $) \pm 0.2(\mathrm{BSR}) \pm 0.8($ th $\left.)\right] \times 10^{-4} \mathrm{fm}^{3}$, which represents a $30 \%$ reduction in the statistical uncertainty.
\end{abstract}

\section{Introduction}

The electric $(\alpha)$ and magnetic $(\beta)$ polarizabilities of the proton have recently been extracted with a statistical uncertainty of $0.35 \times 10^{-4} \mathrm{fm}^{3}$ [5]. This precision is a result of the large number of experimental measurements of $p(\gamma, \gamma) p$ conducted over the last twenty years (see [6] for a review of the experiments). By comparison, in 2012, the uncertainty of the neutron polarizabilities was $1.8 \times$ $10^{-4} \mathrm{fm}^{3}[6]-$ more than five terms larger than for the proton. The primary reason for this discrepancy is the lack of data for Compton scattering from the deuteron which is used to extract $\alpha_{n}$ and $\beta_{n}$.

The previous data for $d(\gamma, \gamma) d$ come from three measurements: Lucas et al. at Illinois [7], Hornidge et al. at Saskatoon [8], and Lundin et al. at Lund [9]. The results of these experiments are summarized in table 1 . The goals of this measurement were therefore

1. to produce more than twenty additional data points with

2. statistical (systematic) uncertainties comparable to those from Illinois (Saskatoon) while

3. the energy bin width $\Delta \mathrm{E}_{\gamma} \approx 8 \mathrm{MeV}$ and

4. overlapping with the low energy data near $70 \mathrm{MeV}$ and extending up to $\sim 115 \mathrm{MeV}$.

Data that meets these goals would effectively double the world database for $d(\gamma, \gamma) d$ and significantly reduce the uncertainties of $\alpha_{n}$ and $\beta_{n}$.

\footnotetext{
a e-mail: lmyers@jlab.org
} 


\section{EPJ Web of Conferences}

Table 1. Comparison of reported results from prior $d(\gamma, \gamma) d$ measurements.

\begin{tabular}{lccccc}
\hline Ref. & $\begin{array}{c}\mathrm{E}_{\gamma} \\
{[\mathrm{MeV}]}\end{array}$ & $\begin{array}{c}\Delta \mathrm{E}_{\gamma} \\
{[\mathrm{MeV}]}\end{array}$ & $\begin{array}{c}\text { Data } \\
\text { Points }\end{array}$ & \begin{tabular}{c}
\multicolumn{2}{c}{$\delta \frac{d \sigma}{d \Omega}$} \\
Stat
\end{tabular} & Syst \\
\hline$[7]$ & 49,69 & $\sim 7$ & 6 & $4-13 \%$ & $\sim 4 \%$ \\
{$[8]$} & 95 & 20 & 5 & $5-10 \%$ & $\sim 5 \%$ \\
{$[9]$} & 55,66 & 10 & 18 & $8-24 \%$ & $7-14 \%$ \\
\hline
\end{tabular}

\section{Experimental Setup}

This measurement was performed at the Tagged Photon Facility at MAX IV (see figure 1). Over the course of two run periods, electron beams with energy of $144(165) \mathrm{MeV}$ were used to produce and tag photons with energies of 65-97(81-115) MeV. The electrons were detected in a focal-plane (FP) hodoscope, which is used for both energy determination and cross-section normalization. The bremsstrahlung photons scattered off the target (either deuterium or carbon) and were detected by the large-volume $\mathrm{NaI}$ detectors. These detectors consist of $\mathrm{NaI}$ cylinders with an outside diameter of $50 \mathrm{~cm}$ and a length greater than $50 \mathrm{~cm}$. The detectors also have a cosmic-ray veto shield and an energy resolution of $\sim 2 \%$ at $100 \mathrm{MeV}$. Further details of the facility and detectors can be found in references $[4,10]$.

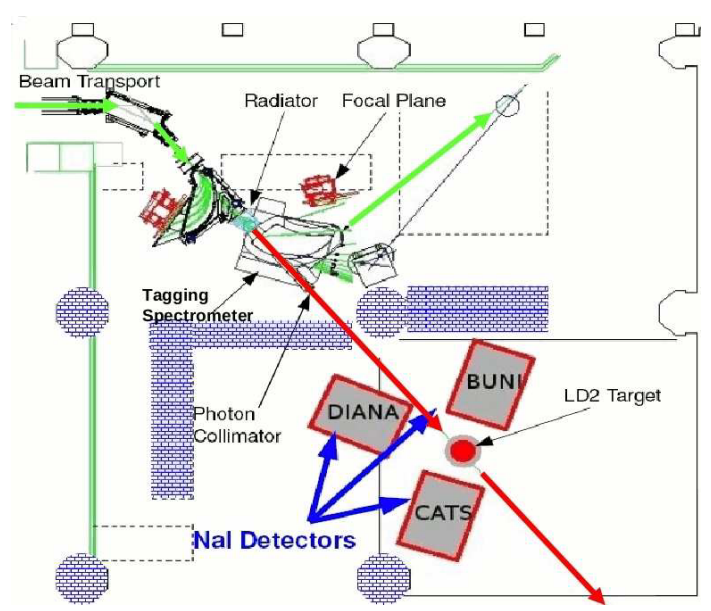

Figure 1. Schematic diagram of the experimental setup at the MAX IV facility. The electron beam impinges on an $\mathrm{Al}$ radiator producing bremsstrahlung photons. The recoil electrons are momentum-analyzed by the tagging spectrometer magnet and detected in the focal-plane hodoscope. The photons interact with the target and scatter into the NaI detectors.

\subsection{Experimental Challenges}

Compton scattering off the deuteron has been difficult to measure precisely. Unlike $p(\gamma, \gamma) p$, the potential for contamination of elastic deuteron data with events from $d\left(\gamma, \gamma^{\prime}\right) n p$ requires (1) that detectors have sufficient energy resolution to separate the elastic photons or (2) that simulations be used to estimate the photon yield from $d\left(\gamma, \gamma^{\prime}\right) n p$. Secondly, the $d(\gamma, \gamma) d$ cross section is $\sim 15 \mathrm{nb} / \mathrm{sr}$ at these 


\section{$21^{\text {st }}$ International Conference on Few-Body Problems in Physics}

energies. Photon intensity at the target is typically on the order of $1-2 \mathrm{MHz} / \mathrm{MeV}$ which necessitates long run periods to collect sufficient statistics while also dealing with a large, untagged background over the same energy range. The approach to handling these issues are to have detectors which good energy resolution and to collect as much data as possible - both of which were accomplished in this experiment.

A third challenge arose during data collection and analysis that is unique to this experiment. As seen in figure 5 of reference [10], the beam suffered from a significant amount of structure. This structure, which was eventually connected to an incomplete filling of the electron ring and the frequency of the extraction shaker, led to large $(\geq 4 \mathrm{MHz})$ instantaneous electron rates in the FP hodoscope. As a result, the cross sections suffered from rate-dependent effects (see sect. 3). The collaboration developed and benchmarked a Monte Carlo simulation $[2,3]$ to calculate the correction factors and estimate the uncertainties.

\section{Data Analysis}

A detailed description of the data analysis and simulations can be found in [10] so only a very brief overview is given here. First, cosmic-ray events were filtered from the raw data. From the reduced data, a missing energy spectra $\left(E_{N a I}-E_{\text {tagged }}\right)$ was filled for events within the prompt timing peak. A similar histogram was also filled for events in the accidental window. A GEANT4 simulation was employed to determine the detector response lineshape for the elastic photons. Finally, the prompt data are fit with the sum of the accidental spectrum (scaled) and the simulated elastic spectrum. From this fit it is possible to subtract the accidental contribution and extract the photon yield.

The elastic photons were integrated over a range from -2 to $+2 \mathrm{MeV}$ in missing energy. This region was deliberately chosen to minimize any contamination from $d\left(\gamma, \gamma^{\prime}\right) n p$. The analysis determined that the contamination is small and statistically consistent with zero, in part because the elastic lineshape describes the data very well over the integrating region $\left(\chi^{2} /\right.$ d.o.f. $\left.\approx 1\right)$. Total yields were on the order of $10^{3}$ events in each energy/angle bin, with statistical uncertainties of $6-16 \%$.

Normalization factors for the cross section included the number of photons incident on the target, the thickness (areal density) of the target, and the solid angle subtended by each NaI detector. For the deuterium target, data was also collected using an empty cell in order to subtract any scattering contribution originating from the cell.

Monte Carlo simulations were used to determine correction factors arising from event filters and the extended geometry of the setup (GEanT4), the effective target thickness, and rate-dependent effects in the FP hodoscope. The systematic uncertainties on these terms was typically on the order of a few percent.

\section{Results}

The analysis steps from the previous section were first applied to data collected using a carbon target. As shown in [4], the cross sections are in excellent agreement with prior measurements. These results indicate that the corrections and their uncertainties are accurately described by the Monte Carlo simulations. As such, it is reasonable to apply the same method to the data collected with the deuterium target.

The final cross sections for $d\left(\gamma, \gamma^{\prime}\right) d$ are shown in figure 2. The error bars shown are statistical only. The systematic (normalization) uncertainties are $\sim 5 \%$ for all the data points. There is an additional uncertainty of 3-4\% correlated for all points at a given angle arising from differences in detection efficiency and solid angle for each $\mathrm{NaI}$. The open symbols correspond to previous measurements at similar kinematics and indicate the consistency of the world data set. 


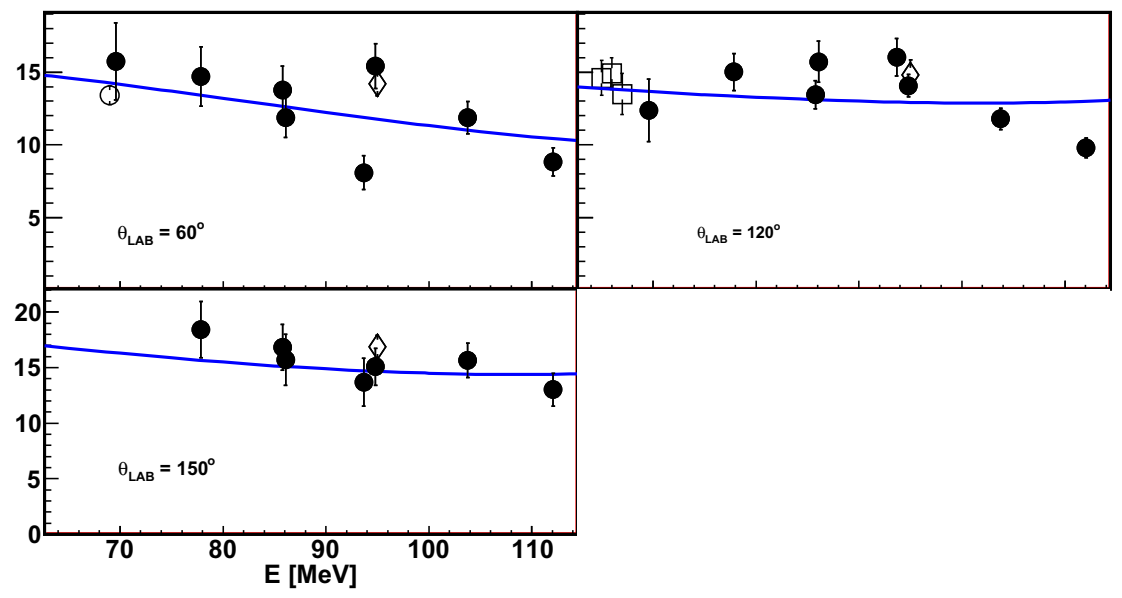

Figure 2. Cross sections from the experiment presented here $(\bullet)$. Open symbols are results from previous measurements, and the solid line represents the best fit from reference [1].

\section{Conclusions}

The results from the COMPTON@MAX-lab collaboration represent the addition of 23 new data points to the world data set with uncertainties comparable to the previous data. The energy range for the Compton scattering cross section has been extended by $\sim 20 \mathrm{MeV}$. As a result of these data, a new extraction of the neutron polarizabilities [1] yields $\alpha_{n}=11.55 \pm 1.25_{\text {stat }}$ and $\beta_{n}=3.65 \mp 1.25_{\text {stat }}$, where the statistical uncertainties are reduced by $\sim 30 \%$. This is an important first step in developing a deuteron Compton scattering database comparable to the one for the proton. Future data from MAX $\mathrm{IV}$, as well as upcoming measurements at $\mathrm{HI} \gamma \mathrm{S}$ and Mainz, should continue to add to the database.

\section{Acknowledgements}

The author acknowledge the support of the COMPTON@MAX-lab collaboration, the staff of the MAX IV Laboratory, and the Data Management and Software Centre, a Danish contribution to the European Spallation Source ESS AB, for generously providing access to their computations cluster. This work was sponsored in part by the US National Science Foundation under Award Number 0855569.

\section{References}

[1] L. S. Myers et al., Phys. Rev. Lett. 113, 262506 (2014).

[2] L. S. Myers et al., Nucl. Inst. Meth. A 729, 707 (2013).

[3] M. F. Preston et al., Nucl. Inst. Meth. A 744, 17 (2014).

[4] L. S. Myers et al., Phys. Rev. C 89, 035202 (2014).

[5] J. A. McGovern, D. R. Phillips, and H. W. Griesshammer, Eur. Phys. J. A 49, 12 (2013).

[6] H. W. Griesshammer, J. A. McGovern, D. R. Phillips, and G. Feldman, Prog. Part. Nucl. Phys. 67, 841 (2012).

[7] M. A. Lucas, Ph.D. thesis, University of Illinois at Urbana-Champaign, unpublished (1994). 


\section{$21^{\text {st }}$ International Conference on Few-Body Problems in Physics}

[8] D. L. Hornidge et al., Phys. Rev. Lett. 84, 2334 (2000).

[9] M. Lundin et al., Phys. Rev. Lett. 90, 192501 (2003).

[10] L. S. Myers et al., Phys. Rev. C 92, 025203 (2015). 